\title{
Nuevas herramientas conceptuales para el análisis de políticas públicas: la literatura sobre difusión y transferencia de políticas
}

\section{Eulalia Rubio Barceló*}

\section{Introducción}

Este artículo pretende ofrecer al lector una visión general de la literatura sobre difusión y transferencia de políticas, así como explorar las potencialidades que ésta ofrece para el análisis de las dinámicas de gobernación en sociedades contemporáneas.

El estudio de procesos de difusión y/o transferencia política es un campo de investigación relativamente reciente, que ha experimentado un auge significativo en los últimos años. Los avances técnicos en el campo de las comunicaciones, la profundización de los procesos de integración regional y el proceso de globalización económica han conllevado una mayor intensificación de este tipo de fenómenos, y con ello un interés creciente en explicar las causas y efectos de la interdependencia entre procesos políticos. El artículo empieza introduciendo al lector al fenómeno de la transferencia política y clarificando los principales conceptos que se manejan en este campo de análisis. Sigue con un breve repaso de la literatura existente, distinguiendo entre dos campos de análisis: los estudios sobre modelos de difusión y los estudios sobre transferencia de políticas. Finalmente, el tercer apartado discute las limitaciones y potencialidades que ofrece el análisis de la difusión y de la transferencia política para el estudio de las dinámicas de gobernación en sociedades contemporáneas.

\section{La transferencia de políticas: un fenómeno en expansión}

La transferencia de políticas se define, en términos generales, como el proceso mediante el cual el conocimiento sobre políticas, programas o instituciones de un sistema político influye, de una forma más o menos significativa, en la adopción de políticas, programas o instituciones similares en otro sistema político. En términos más politológicos, podríamos decir que los estudios sobre difusión y/o transferencia política se ocupan del análisis de la interdependencia entre procesos políticos; esto es, situaciones en las que la decisión tomada por un gobierno (e.g. la adopción de una nueva política) influye, de un modo u otro, en la toma de decisiones posterior de otro gobierno formalmente independiente.

La interdependencia entre procesos políticos no es un fenómeno estrictamente contemporáneo; desde la antigüedad, la toma de decisiones políticas se ha sustentado en parte en el conocimiento sobre las estructuras políticas y las acciones desarrolladas en otros países, imperios o reinados, y la imitación o emulación ha sido un recurso frecuente. En las últimas décadas, sin embargo, una serie de factores más o menos relacionados con los procesos de globalización económica, política y social han intensificado la interdependencia entre territorios 
nacionales y han favorecido las dinámicas de transferencia (Evans y Davis, 1999, Stone, 1999, Dolowitz y MarSh, 2000).

En primer lugar, los procesos de globalización económica y social han intensificado la interdependencia funcional entre países. Cada vez más, las decisiones tomadas en un territorio producen efectos importantes económicos y sociales en otros territorios. Ello incentiva las prácticas de imitación o emulación entre países, sea para evitar externalidades negativas (movilidad del capital) o para aprovechar externalidades positivas derivadas de la coordinación (e.g., introducción de las mismas regulaciones sobre estándares de producción, niveles de contaminación, etc.) (HoBERG, 1991) ${ }^{1}$. Por otro lado, las dinámicas de cambio social son cada vez más de alcance global, los gobiernos se enfrentan al mismo tipo de problemas (e.g., inmigración, desempleo) y ello incentiva la transferibilidad de programas y políticas.

En segundo lugar, los avances técnicos en el campo de las comunicaciones y la profundización de los procesos de integración regional han facilitado el intercambio de ideas e información a nivel transnacional (KoHLER-KoCH, 2002). Cada vez es más fácil disponer de información sobre lo que hacen otros países en diferentes campos de intervención, y cada vez más técnicos y élites políticas de diferentes gobiernos establecen contactos, participan en conferencias internacionales y realizan visitas oficiales a otros países para conocer sobre la implantación de programas específicos.

Finalmente, los procesos de difusión y transferencia política han sido promovidos por organizaciones de alcance internacional (e.g., Unión Europea, OMC, NAFTA). Con la voluntad de evitar efectos positivos o negativos de la interdependencia, estas organizaciones han institucionalizado mecanismos de comunicación tendentes a facilitar la difusión horizontal o entre países, y han promovido la armonización de políticas a través de prácticas de persuasión (e.g., actuando como 'ideational entrepreneurs', generando nuevos conceptos o políticas y favoreciendo su diseminación de diferentes formas) o de semi-coerción (e.g., estableciendo la adopción de cambio institucional o una determinada medida política como prerrequisito para la obtención de ayudas financieras).

\section{Clarificando conceptos: difusión (policy diffusion), transferencia (policy transfer), convergencia (policy convergence) y aprendizaje político (policy learning)}

Hasta aquí se han utilizado los conceptos 'difusión' y 'transferencià como sinónimos. Sin embargo, la mayor parte de la literatura distingue en el uso de los términos 'difusión' (policy diffusion) y 'transferencia' (policy transfer). El término difusión suele utilizarse para definir un fenómeno macro, el proceso general de diseminación de una innovación política (e.g., un nuevo enfoque, un nuevo programa, una reforma institucional) en varias jurisdicciones que forman parte de un mismo contexto nacional o internacional. El termino transferencia, en cambio, se utiliza para denotar un fenómeno de naturaleza 'meso', el proceso (o los procesos) de decisión política que conlleva la exportación de una política, programa o enfoque de un sistema político a otro ${ }^{2}$. Aparte de la diferencia en la dimensión de análisis (macro o meso), difusión y transferencia se distinguen por las diferentes presunciones que conllevan con respecto a la relevancia de los factores estructurales y agenciales en los procesos de interdependencia entre procesos políticos. Así, transferencia es un término que se asocia con la existencia de una actividad intencional (e.g., un alguien que actúa como agente transferidor), mientras que el término difusión evoca la idea de contagio, y asume un rol más pasivo de los actores políticos en los procesos de exportación de políticas (STONE, 1999, 2003).

Otro término que se encuentra con frecuencia en la literatura sobre transferencia y difusión de políticas es el de convergencia en políticas (policy convergence). El término convergencia denota la existencia de un patrón general de creciente similitud en la organización económica, social o política de distintas sociedades, más o menos asociado con procesos de industrialización, regionalización o globalización (STONE, 2003, p.5). Más específicamente, 'convergencia en políticas' suele utilizarse para referirse a la idea que las sociedades industriales avanzadas tienden cada vez más a enfrentarse a problemas similares y a resolver éstos de forma parecida (BENETT, 1991). Difusión y convergencia son fenómenos conectados entre sí, pero no significan exactamente lo mismo. La difusión de políticas conlleva una dinámica de convergencia; sin 
embargo, la convergencia en políticas no siempre es debida a un proceso de difusión. Los procesos de difusión se producen cuando existe una interdependencia en los procesos de decisión política; cuando la decisión tomada por un gobierno (e.g., la adopción de una nueva política) influye, de un modo u otro, en la toma de decisiones posterior de otro gobierno (SimMONS y ElKINS, 2003). La convergencia en políticas, en cambio, es un fenómeno más amplio: otros factores pueden explicar el proceso de convergencia en las políticas (e.g., procesos de armonización o coordinación negociada, existencia de cambios estructurales similares que dan lugar al desarrollo de políticas parecidas en distintos países).

Finalmente, el concepto de policy learning o aprendizaje político también aparece con frecuencia en la literatura sobre transferencia de políticas. Policy learning se refiere al proceso de cognición y de redefinición de intereses en base a nuevo conocimiento adquirido que afecta las creencias fundamentales y las ideas que fundamentan una determinada política (HALL, 1993, citado en STONE, 2003). Es, por lo tanto, un concepto relacionado con el modo en que los actores políticos utilizan la información en la toma de decisiones. Tradicionalmente, ha habido una tendencia en la literatura a conceptualizar la transferencia de políticas como un proceso de aprendizaje político. Más concretamente, se suele definir la transferencia como un proceso de lesson-drawing (Rose, 1991), esto es, un proceso de recogida de información, evaluación y adaptación de las experiencias desarrolladas en otros países con el objetivo de mejorar la eficacia de las propias políticas. Sin embargo, no todos los procesos de transferencia son el resultado de un aprendizaje político. En primer lugar, la importación de políticas o instituciones no siempre es el resultado de una elección voluntaria de los actores domésticos; puede ser inducida por la presión directa de otros actores (e.g., cuando las organizaciones internacionales imponen la introducción de una medida como condición para recibir una ayuda financiera) o por la presión indirecta del entorno (e.g., la necesidad de conformar las propias políticas o instituciones a las de los gobiernos colindantes, a fin de evitar externalidades negativas derivadas de la falta de armonización). En segundo lugar, la visión de la transferencia como un proceso de 'lesson-drawing' se basa en la asunción de que la acción política es instrumental (e.g., orientada a la solución de problemas). Sin embargo, la importación de una institución, política o programa puede tener también una finalidad simbólica o legitimadora (GILARDI, 2003, WEYLAND, 2003).

\section{La literatura sobre difusión y transferencia de políticas}

Las diferencias conceptuales entre 'difusión' y 'transferencia' han dado lugar al desarrollo de dos perspectivas teórico- metodológicas en el estudio de las dinámicas de exportación/importación de políticas. Por un lado, el análisis de modelos de difusión, de tipo cuantitativo, inspirado en los modelos y teorías sociológicas sobre difusión de innovaciones entre individuos u organizaciones (e.g., Rogers, 1995, PALloli, 1998). Por otro lado, los estudios sobre procesos de transferencia, de tipo cualitativo, que emergen como un campo de investigación específico dentro del análisis del proceso político. Como veremos a continuación, estas dos perspectivas de análisis difieren en cuanto al nivel de análisis (macro o meso), las preguntas de investigación y las presunciones de partida en la explicación de los procesos de interdependencia política (más estructuralistas en el primer caso, más agenciales en el segundo).

\subsection{El análisis de modelos de difusión}

El análisis de modelos de difusión tiene como objetivo describir y explicar el patrón temporal y espacial de difusión de una innovación política en varias jurisdicciones que forman parte de un mismo contexto (nacional o internacional). Este tipo de estudios se empezaron a desarrollar en los años 60 en los Estados Unidos, inspirados por los modelos y teorías existentes en sociología y en teoría de las organizaciones sobre difusión de innovaciones entre individuos u organizaciones (Rogers, 1995, PaLloli, 1998). Los primeros estudios se centraron en el análisis de procesos de difusión intra-nacional, entre Estados federales (e.g. WalKer, 1969, GraY, 1973) o municipios (Bingham et al., 1978). A partir de los noventa, sin embargo, el análisis de difusión de políticas se ha interesado básicamente en procesos de difusión inter-estatal (e.g., BENNET, 1997, KERN et al., 2001, Weyland, 2002, Meseguer, 2003, Gilardi, 2003).

El análisis de modelos de difusión es un análisis de tipo 'macro'; se interesa en explicar un fenómeno de alcance transnacional (la difusión de una innovación política en varios territorios), sin prestar demasiada atención a lo que ocurre a nivel doméstico (e.g, cómo y quién decide introducir la innovación política y el impacto que ello produce). Como resultado de ello, tiende a enfatizar la importancia de los factores estructurales (e.g., localización geográfica, características socio-económicas del país,..) en la explicación de los procesos de difusión. La mayoría de estudios analizan la difusión de programas específicos o de ciertas reformas institucionales, e.g.: la introducción de una legislación sobre la libre elección de escuelas (e.g., MinTrOM y VERGARI, 1998), la creación de una lotería nacional (BERRY y BERRY, 1990), la adopción de un sistema de etiquetaje ecológico (KERN et al., 2001), la creación de una agencia de regulación independiente (GILARDI, 2003), la puesta en marcha de procesos de privatización o liberalización (MesegUer, 2003, Simmons y ELKIN, 2003) o la introducción de nuevos instrumentos de control burocrático (e.g., BENNET, 1997). En cuanto a las preguntas 
de investigación, éstas han ido cambiando a lo largo del tiempo. La introducción de nuevas técnicas econométricas ha ido permitiendo un mayor refinamiento de las preguntas de investigación y el desarrollo de modelos explicativos más complejos. En este sentido, es posible distinguir entre tres generaciones de estudios sobre difusión de políticas.

La primera generación de estudios sobre difusión se orientaba a averiguar el impacto relativo de la difusión en un proceso de convergencia de políticas. Para ello, los estudios se encaminaban a testar dos hipótesis alternativas; la hipótesis de la difusión (según la cual el proceso de convergencia se debía a procesos de imitación entre países) y la hipótesis nula (según la cual la convergencia era el resultado de procesos similares de cambio estructural experimentados de forma paralela, pero independiente, en distintos territorios). Utilizando técnicas estadísticas tradicionales (análisis de regresión -Collier y MESSICK, 1975-, análisis factorial -WALKER, 1969-), las evidencias empíricas que aportaban eran poco robustas: basados en datos agregados, la práctica habitual era inferir la existencia de un proceso de difusión a partir de la evidencia residual o, más en general, de la inconsistencia entre el patrón de difusión real y aquel predicho por la hipótesis nula.

A principios de los noventa, la introducción de una nueva técnica estadística en el análisis de la difusión (el event history analysis) permite desarrollar modelos explicativos más complejos, que incorporan a la vez el impacto de los factores domésticos y de los factores de difusión en el estudio de procesos de convergencia. Ello da lugar a una nueva generación de estudios, cuyo interés principal ya no es aislar el impacto relativo de la difusión sino identificar los factores estructurales (e.g., localización geográfica, estructura socio-económica del país) que explican el patrón temporal y espacial de difusión de una determinada innovación política (e.g., BERRY y BERRY, 1990, 1998; MinTrOM y VERGARI, 1998).

Finalmente, en los últimos años ha aparecido una nueva generación de estudios que, utilizando técnicas econométricas aún más complejas, han permitido desarrollar modelos en los que se incorpora por primera vez el factor agencial. A diferencia de los estudios previos, en los que el factor agencial era simplemente no tenido en cuenta o bien era modelizado como una constante (e.g,. se presumía que la difusión era el resultado de un proceso de aprendizaje político), esta nueva generación de estudios parte de la presunción que existen diferentes motivaciones o preferencias que pueden conducir a un proceso de imitación o copia y, a nivel macro, a un proceso de difusión. Modelizando éstas en términos de 'mecanismos de difusión', el objetivo de estos nuevos estudios es identificar qué mecanismos explican mejor un determinado patrón espacial y temporal de difusión ${ }^{3}$.

\subsection{La literatura sobre transferencia de políticas}

La literatura sobre transferencia de políticas tiene como objetivo describir y explicar el proceso de decisión interna que acompaña un proceso de transferencia. En palabras de Dolowitch y MARSH, se interesa en explicar cómo, cuándo y por qué "el conocimiento sobre políticas, programas o instituciones en un tiempo y/o espacio determinado son utilizados en el desarrollo de políticas, programas o instituciones en otro tiempo o lugar" (1996: 344). Si bien existen ciertos precedentes (e.g., HeCLO, 1974, Hall, 1989), el análisis de la transferencia política se desarrolla sobretodo en los años noventa, como una subcorriente de investigación en el ámbito de la política comparada y de las relaciones internacionales. Ejemplos de este tipo de estudios son los trabajos de Rose (1991), Dolowitz (1997) y BENNETT (1991, 1997).

La literatura sobre transferencia de políticas es mucho más caótica y heterogénea que la literatura sobre difusión. Tal como señalan EVANS y DAVIS (1999), se caracteriza por su multidisciplinariedad y la ausencia de un marco teórico y metodológico común ${ }^{4}$. En los últimos años ha habido un esfuerzo en desarrollar marcos heurísticos comunes (DOLOWITZ y MARSH, 1996, 2000, Evans y DaVIS, 1999, Stone, 1999). A partir de estas contribuciones, es posible identificar las principales características de este campo de investigación.

La principal característica del análisis de transferencia es su enfoque 'meso'. El objetivo del investigador es explicar el proceso de decisión política interna que conlleva la exportación de una política, programa o enfoque de un sistema político a otro. El objeto de investigación puede ser muy diverso; desde programas $o$ instituciones específicas hasta aproximaciones o ideas, actitudes y 'lecciones negativas' (DOLOWITZ y MARSH, 2000). En cuanto a la metodología, la mayoría son estudios de un caso singular, de tipo cualitativo. La evidencia empírica que se usa para demostrar la existencia de una transferencia proviene, básicamente, de entrevistas con los actores participantes y de fuentes documentales varias (e.g., prensa, intervenciones en debates parlamentarios, informes oficiales, legislación).

Los estudios sobre transferencia de políticas enfatizan la importancia del factor agencial. Tal como señalan EvaNs y DAVIS (1999), el elemento intencional se considera un elemento definitorio del proceso de transferencia. Más específicamente, la mayor parte de estudios se sustentan en la presunción implícita de que la transferencia es el resultado de un proceso de aprendizaje político, y que la élite política del país receptor es el actor principal en el proceso de importación de las políticas. El objetivo final de las investigaciones, en este sentido, es identificar los factores contextuales que explican la decisión de la elite política de importar un determinado programa o política desarrollado en otro país. La mayoría de estudios 
adoptan un enfoque clásico para el análisis del proceso decisional, basado en el modelo secuencial o por etapas del proceso político, y explican la decisión de importar a partir de modelos explicativos del cambio en la agenda política, como el modelo de las múltiples corrientes de KINGDON ${ }^{5}$. En los últimos años, ha habido un cierto interés en adoptar nuevos enfoques analíticos para el estudio de procesos de transferencia. En este sentido, algunos autores (e.g., STONE, 1999, Evans y Davis, 1999) han sugerido la posibilidad de aplicar el análisis de redes políticas, y más específicamente modelos explicativos de la transmisión de información a escala intergubernamental, como el modelo de Advocacy Coalitions de SABATIER ${ }^{6}$ o el modelo de las Epistemic Communities de HAAS ${ }^{7}$. La adopción de estos modelos, se argumenta, permitiría concebir la transferencia como un fenómeno intergubernamental en el que participan los decisores políticos del país importador, pero también otros actores públicos y privados que operan a escala transnacional.

\section{Problemas teóricos y metodológicos de los estudios sobre difusión y sobre transferencia de políticas}

A pesar del desarrollo significativo que han experimentado tanto la literatura sobre difusión como la literatura sobre transferencia de políticas, ambas corrientes de investigación siguen adoleciendo de ciertas limitaciones teórico-metodológicas para el análisis y la explicación de los fenómenos de interdependencia política.

A nivel teórico-conceptual, el principal problema que presentan ambas corrientes es su desconexión con los grandes debates o cuestiones que orientan la investigación en ciencia política. Ésta es más marcada en el análisis de la transferencia política. La mayoría de estudios sobre la transferencia son empírico-descriptivos, se orientan principalmente a demostrar si se ha producido o no un proceso de transferencia sin que el proceso de búsqueda y análisis de información esté guiado por un marco conceptual definido, con hipótesis de investigación específicas sobre qué factores han motivado el proceso. En cuanto al análisis de modelos de difusión, éste sí se suele organizar a partir de un marco teórico-conceptual definido. No obstante, la formulación de preguntas e hipótesis de investigación ha estado muy condicionada por las limitaciones derivadas del uso de métodos cuantitativos. Más concretamente, dos limitaciones se derivan del hecho de utilizar la estadística como herramienta de análisis. En primer lugar, el uso de técnicas cuantitativas implica la adopción de una lógica nomotética o de regularidades causales para la explicación de los fenómenos sociales (SMELSER, 1976). En el análisis de difusión, implica presumir que las unidades de análisis que componen el proceso general de difusión (e.g., los múltiples procesos de decisión internos que dan lugar a un proceso general de difusión) son explicables a partir de un único modelo explicativo. En segundo lugar, la propia naturaleza del análisis estadístico limita el conocimiento sobre el proceso de decisión política que subyace en un proceso general de difusión. Como se ha mencionado, los avances econométricos han permitido en los últimos años refinar la construcción de las variables explicativas, con el objeto de esclarecer cómo el conocimiento de las políticas desarrolladas en otros países influye en la toma de decisiones interna. A pesar de ello, las nuevas variables siguen siendo variables proxies para medir lo que ocurre a nivel 'meso'. En otras palabras: no nos aportan información directa sobre las dinámicas políticas que se producen a nivel doméstico (e.g., cómo se introduce la información en el proceso político, quién la utiliza y cómo se utiliza). Además, el análisis de difusión no permite analizar y comparar el contenido específico de la transferencia en diferentes territorios, o el impacto que ésta produce en términos de cambio político. La variable dependiente de la investigación, la acción de la transferencia, se conceptualiza como un acto de 'todo o nada' y se operacionaliza como una variable dicotómica, en términos de adopción o no de una legislación o de una determinada reforma institucional $^{8}$ (DOLOWITZ y MARSH, 2000).

La otra gran limitación que presenta el estudio de la interdependencia política es el hecho de haberse desarrollado de forma separada, desde dos perspectivas analíticas distintas, cada una de ellas basada en el uso exclusivo de un tipo de metodología (cualitativa en el análisis de la transferencia, cuantitativa en el análisis de difusión). Como hemos ya señalado, el uso exclusivo de la metodología cuantitativa impone limitaciones importantes en el análisis de difusión. De forma similar, los estudios sobre transferencia de políticas presentan importantes problemas de validación empírica que se derivan del hecho de basarse en el análisis cualitativo de un único caso de estudio. 


\section{Potencialidades del análisis de difusión y/o transferencia de políticas: una nueva herramienta para el estudio del cambio en las políticas}

Las limitaciones que presentan ambas perspectivas de análisis no deben ofuscar las posibilidades que ofrece el análisis de la interdependencia política para el estudio de las dinámicas de gobernación en sociedades contemporáneas.

A nivel teórico-conceptual, su principal contribución es la de resolver el problema de Galton; es decir, quebrantar la clásica presunción de independencia entre los casos de análisis que sustenta tradicionalmente los estudios sobre política comparada. Si bien esta presunción sigue siendo válida como punto de partida para el investigador, el análisis de difusión y/o transferencia de políticas se vislumbra como una alternativa para la explicación de procesos de formación y/o procesos de cambio en las políticas en ámbitos o sectores de intervención en que existen indicios suficientes de interdependencia política (e.g., regulación de sectores económicos altamente globalizados, políticas con alto grado de europeización, problemas sociales que trascienden las fronteras nacionales, etc..). En un contexto de globalización creciente, ello parece ser cada vez más la regla y no la excepción.

A nivel más analítico, el estudio de la interdependencia política puede contribuir a mejorar el conocimiento sobre el proceso político. Más específicamente, éste puede conectarse con dos grandes preguntas o cuestiones que orientan la investigación en ciencia política. Por un lado, el impacto de los factores exógenos (e.g., globalización, europeización) en la toma de decisiones a nivel nacional. Por otro lado, el rol que juega la información en la toma de decisiones políticas.

La primera cuestión ha suscitado importantes debates en los últimos años. En el ámbito de la economía política y del Estado del Bienestar, el impacto de la globalización se ha convertido en uno de los principales objetos de discusión. En general, los autores se han posicionado en términos de 'globalistas' y no 'globalistas', en una especie de continumm según el mayor o menor grado de autonomía o capacidad agencial reconocido a los actores políticos. Esta visión del debate, sin embargo, supone concebir la globalización en términos simplistas, como un proceso que limita o constriñe la capacidad de maniobra política. Los estudios sobre difusión y/o de transferencia de políticas permiten concebir la globalización de una forma más compleja, como un proceso político, social y cultural que es susceptible de influir en las dinámicas políticas internas de formas muy diversas: de forma directa, limitando ciertas alternativas de acción política, pero también de forma indirecta, favoreciendo el intercambio de información entre países y potenciando dinámicas de imitación o emulación. De forma similar, los estudios sobre interdependencia política pueden contribuir a mejorar el conocimiento sobre los procesos de europeización. En los últimos años, ha habido un interés creciente en analizar y comparar cómo la dimensión europea ha penetrado en las instituciones, políticas y modos de gobernación de los países miembros. En general, el proceso de europeización se ha conceptualizado en términos de adaptación o ajuste a las regulaciones europeas (e.g., KNILL, 1998, BORZEL, 1999). Sin embargo, la europeización es un fenómeno más amplio, que tiene lugar no solamente a través de la imposición de regulaciones sino también a través de la difusión de ideas, conceptos o nuevos enfoques de intervención. (RADAELLI, 2000, KNILL y LeHMKUHI: 2002). Según algunos autores, la Comisión Europea es el promotor directo de estos procesos de difusión; actúa como ideational entrepreneur, generando nuevos conceptos o políticas y favoreciendo su diseminación de diferentes formas (e.g. evaluación, publicación y difusión de resultados, financiación de nuevos programas). Otros, en cambio, enfatizan la importancia del intercambio de información y experiencias a través de redes de actores o policy networks que se desarrollan en la formulación e implementación de políticas europeas (HEINELT y Hoogue, 1996, KoHLER-KoCH, 2002). El análisis de difusión y/o transferencia puede ayudar a esclarecer mediante qué mecanismos se produce la difusión de políticas a nivel europeo, e.g., si ésta se produce principalmente como resultado de una acción intencionada de la Comisión (e.g., difusión vertical) o es más bien el resultado del intercambio de información entre actores gubernamentales que operan en redes transeuropeas (e.g., difusión horizontal).

La segunda cuestión está también cada vez más presente en los análisis de políticas públicas. Preguntarse sobre el modo en que se incorpora la información en la toma de decisiones no es más que preguntarse sobre la racionalidad de la acción política. En este sentido, es posible distinguir dos debates o elementos de discusión en el estudio del proceso político; el debate sobre racionalidad absoluta $v$ s. racionalidad limitada y el debate sobre racionalidad técnica vs racionalidad política (WEYLAND, 2002).

En relación con el primero, los autores discrepan con respecto a la cantidad y calidad de información técnica utilizada en la toma de decisiones políticas. Según algunos, es útil presumir que los decisores públicos disponen de la información suficiente en la toma de decisiones; antes de decidir recogen la información necesaria para dibujar las diferentes alternativas de 
acción existentes, evalúan sus costes y beneficios y adoptan aquella que parece más eficiente (racionalidad absoluta). Para otros, los presupuestos de racionalidad absoluta son irreales, y por tanto poco explicativos de lo que ocurre en realidad. De acuerdo con éstos, es necesario rebajar los presupuestos hacia concepciones de 'racionalidad limitada' (LINDBLOM, 1991); es decir, presumir que los decisores actúan en entornos caracterizados por la falta de información y tiempo; que, en la mayoría de casos, no disponen de información sobre todas las alternativas de acción disponibles y que suelen adoptar aquella que parece más satisfactoria.

El otro punto de discusión es relativo al modo en que la información es utilizada. Tradicionalmente, el analista de políticas públicas presumía que los actores políticos, si bien condicionados por factores de tipo contextual (e.g., ciclo electoral), cuando actuaban como formuladores de políticas adoptaban una actitud 'problem-solving' u orientada a la solución de problemas. En la actualidad, sin embargo, el presupuesto de la racionalidad instrumental o técnica es raramente sostenido. El análisis de políticas públicas toma en consideración la existencia de otras lógicas de actuación que influyen en la toma de decisiones, y que pueden llegar a predominar sobre la lógica técnica. Ello es así por dos razones. Primero, porque en un mundo complejo y cambiante, raramente los actores políticos tienen unas preferencias específicas formadas sobre el curso de acción a seguir, y raramente disponen de tiempo e información para desarrollarlas. Segundo, porque en el mundo político real no existe una distinción nítida entre la esfera de las políticas públicas (policies) y la esfera del comportamiento político (politics). Los actores políticos tienen en cuenta ambas lógicas de actuación (técnica y política) en todas las decisiones que toman, sean o no sean propiamente en el ámbito de la formulación de políticas.

Los análisis de difusión y transferencia pueden contribuir a esclarecer el tipo de racionalidad predominante (absoluta o relativa, técnica o política) en la toma de decisiones políticas. El análisis de modelos de difusión ha aportado ya interesantes resultados a este respecto, mediante su distinción entre diferentes mecanismos de difusión (aprendizaje, emulación, difusión simbólica). Sin embargo, dada su naturaleza cuantitativa, la evidencia empírica que aportan estos estudios es limitada y, en algunos casos, no del todo frable (la importancia relativa de una u otra lógica se mide en función de su impacto a nivel 'macro', predicho a partir de una serie de presunciones). Para superar estas limitaciones, el análisis de transferencia podría ser de gran ayuda. El estudio de caso podría servir para confirmar o rechazar la evi- dencias empíricas aportadas por el análisis cuantitativo. Podría también servir para refinar las hipótesis sobre el modo en que la información sobre experiencias ajenas se incorpora en el proceso político (BENNETT, 1991).

\section{Conclusiones}

Cualquier intento de estudiar y explicar un fenómeno político en el mundo contemporáneo debe tener en cuenta la existencia de situaciones de interdependencia política; esto es, situaciones en las que la decisión tomada por un gobierno (e.g., la adopción de una nueva política) influye, de un modo u otro, en la toma de decisiones posterior de otro gobierno formalmente independiente.

El análisis de la interdependencia política se ha desarrollado significativamente en los últimos años, desde dos perspectivas distintas. Por un lado, el análisis de modelos de difusión, de tipo cuantitativo, centrado en explicar la dimensión 'macro' del fenómeno (difusión o diseminación de una política). Por otro lado, el estudio sobre procesos de transferencia política, de tipo cualitativo y centrado en explicar la dimension 'meso' del fenómeno (el proceso de decisión política interna que conlleva la exportación de una política, programa o enfoque de un sistema político a otro). A pesar de estudiar un mismo fenómeno, estas dos aproximaciones se han desarrollado de forma separada la una de la otra, y sólo en contadas excepciones los autores han intentado integrar los avances teóricos y metodológicos de ambas para el estudio de un mismo proceso de difusión (e.g., BENNETT, 1997, MinTROM y VerGari, 1998).

Por otro lado, en ambos casos ha habido poco esfuerzo por insertar el análisis empírico con grandes cuestiones o debates teóricos que orientan la investigación en el ámbito de las ciencias políticas. En este sentido, el artículo sugiere dos grandes debates o líneas de investigación, especialmente relevantes en el ámbito de las políticas públicas, en las que insertar el análisis de difusión y transferencia de políticas. Por un lado, la investigación sobre el impacto de factores exógenos (e.g., globalización, europeización) en las dinámicas políticas domésticas. Por otro lado, la investigación sobre el rol que juega la información y el conocimiento en la toma de decisiones políticas. 
* Investigadora del Instituto Universitario Europeo (Florencia).

${ }^{1}$ En el ámbito estrictamente económico, algunos autores argumentan que el resultado de ello ha sido una tendencia a la armonización 'por debajo' ('race to the bottom): dada la movilidad del capital, los Estados se han visto forzados a introducir las mismas reformas políticas liberalizadoras (e.g., en el ámbito de la política fiscal, macroeconómica, laboral) introducidas por sus directos competidores, a fin de mantener o atraer inversiones (e.g., Daniels: 1991, PORTER: 1999, citados en SimMOnS y ELKINS: 2003). La mayoría de autores, sin embargo, coinciden en observar que la interdependencia entre economías estatales es mayor, y que la imitación se ha convertido en una práctica habitual en el ámbito de la gobernación de la economía, pero ponen en duda que el resultado final sea inevitablemente la armonización de mínimos.

${ }^{2}$ Nótese que el primer termino (difusión) es más restrictivo en su alcance denotativo que el segundo (transferencia). Mientras todo proceso de difusión conlleva varios procesos de transferencia, un proceso de transferencia no necesariamente debe producirse en el marco de un proceso general de difusión de políticas.

${ }^{3}$ Aunque existen diferencias, la mayoría de autores suelen distinguir entre cinco tipo de mecanismos de difusión: difusión por presión externa, difusión por aprendizaje, difusión por emulación o mimetismo, difusión por interdependencia funcional y difusión por imitación simbólica (GILARDI: 2003, WEYLAND: 2003).

4 Estos mismos autores identifican hasta cinco enfoques analíticos (international structure and agency, the epistemic community approach, domestic structure and agency, policy network analysis and formal policy transfer analysis) desde los que se ha desarrollado el análisis de la transferencia política.

5 El modelo de las múltiples corrientes de KINGDON (1996) explica los cambios en la agenda política como el resultado de la interacción de tres corrientes (e.g., combinaciones de actores y procesos) que fluctuan de forma relativamente independiente; una dominada por los debates sobre la naturaleza y definición de los problemas (problem stream), una dominada por las ideas sobre políticas y sobre el modo de resolver los problemas (policy stream) y una tercera dominada por los cambios en el contexto político-electoral (politics stream). Según este modelo, los grandes cambios en la agenda política se producen cuando estas tres corrientes se acoplan temporalmente, creando 'ventanas de oportunidad' temporales que son aprovechadas por parte de los promotores políticos para incorporar un nuevo tema en la agenda política.
${ }^{6}$ El modelo de las Advocacy Coalitions, desarrollado por SABATIER y JenKins-Smith (1993) pretende explicar procesos de cambio en las políticas en periodos temporales de una década o más. Este enfoque se centra en la interacción entre diferentes coaliciones de intereses ('advocacy coalitions') que operan en el marco de un mismo subsistema político y que compiten para imponer una determinada visión del problema o de la política en cuestión. Una coalición de interés se define como un conjunto de actores provenientes de diferentes instituciones (publica o privada, nacional o inernacional) que comparten una serie de creencias e ideas sobre la política en cuestión. Una de las ventajas del modelo es que adopta como unidad de análisis el subsistema político, y no una específica estructura gubernamental o un programa específico. Otra ventaja es que enfatiza la importancia de la información y las ideas como factores causales del cambio en las políticas.

7 El modelo de las 'comunidades epistémicas', propuesto por ADLER y HAAS, es parecido al modelo de SABATIER. Al igual que éste, asume la existencia multiples comunidades políticas, compuestas por actores de diferente tipo y diferente adscripción (nacional o internacional) que compiten entre sí con el objetivo de imponer una determinada visión del problema en un subsistema político. El modelo de las comunidades epistémicas se ha utilizado sobretodo en el ámbito de las relaciones internacionales para explicar el predominio de un determinado paradigma cognitivo en un área de intervención. A diferencia del modelo de SABATIER, no presta especial atención a las dinámicas de cambio, y enfatiza aún más que el modelo de SABATIER la importancia del conocimiento compartido como elemento definidor de la comunidad política (PETERS: 1998).

8 El estudio de DoLowitz sobre la importación de las experiencias americanas de welfare-to-work al Reino Unido (DoLowITZ: 1997) es, en cierta medida, un ejemplo paradigmático. El estudio explica la decisión del gobierno inglés de introducir las experiencias de welfare-to-work americanas al Reino Unido. A partir de un análisis documental y de entrevistas, el autor concluye que la decisión, tomada a mediados de los noventa, fue el resultado de una combinación de cuatro factores: la percepción de insatisfacción con el funcionamiento de la política preexistente, el crecimiento alarmante de la tasa de desempleo, el contexto electoral y la existencia de una ola generalizada de procesos de reforma y modernización del mercado laboral en Europa (1997: 24).

\section{Bibliografia}

Bennett, Colin J. 1991. "How States Utilize Foreign Evidence". Journal of Public Policy 11, 1: 31-54.

BennetT, Colin J. 1997. "Understanding Ripple Effects: The Cross-National Adoption of Policy Instruments for Bureaucratic Accountability". Governance: An International Journal of Policy and Administration 10, 3: 213-233.

BerRY, Frances Stokes y BerRY, William D. 1990. "State Lottery Adoptions as policy innovations: An Event History Analysis". The American Political Science Review 84, 2: 395-415.

Berry, Frances Stokes y BerRY, William D. 1999. "Innovation and Diffusion in Policy Research", en Theories of the Policy Process, ed. Sabatier, Paul A. Colorado: Westview Press.

Bingham, Richard D. 1978. "Innovation, Bureaucracy and Public Policy: A Study of Innovation Adoption by Local Government". The Western Political Quarterly $31,2: 178-205$

Borzel, Tanja. 1999. "Towards Convergence in Europe? Institutional Adaptation to Europeanisation in Germany and Spain", Journal of Common Market Studies, 37, 4: 573-96.

Chapman, Bruce y GreenawaY, David. 2003. "Learning to Live with Loans? Policy Transfer and the Funding of Higher Education”. Paper presentado en la conferencia Political Economy of Policy Transfer, Learning and Convergence, Tulane University, Abril 2003.
Collier, David y Messick, R.E. 1975. "Prerequisits versus Diffusion: Testing Alternative Explanations of Social Security Adoption". American Political Science Review 69:1299-1315.

Deacon, Bob, Hulse, Michelle y StubBs, Paul. 1997. Global Social Policy: International Organizations and the Future of Welfare. London:Sage.

Dolowitz, David and David MarSH. 1996. "Who Learns What from Whom: a Review of the Policy Transfer Literature”. Political Studies 44: 343-357.

DolowITZ, David. 1997. "British Employment Policy in the 1980s: Learning from the American Experience". Governance: An International Journal of Policy and Administration 10, 1: 23-42.

DolowiTZ, David y MaRSH, David. 2000. Learning from Abroad: The Role of Policy Transfer in Contemporary Policy-Making. Governance: An International Journal of Policy and Administration 13, 1: 5-24.

Duncan, Alan, Pearson, Mark and ScholZ, John Karl. 2003. "Is there and emerging consensus in making work pay policies?", Paper presentado en la conferencia Political Economy of Policy Transfer, Learning and Convergence, Tulane University, Abril 2003.

ESPING ANDERSEN, Gosta. 1999. Social Foundations of Post-Industrial Economies. New York/Oxford: Oxford University Press.

EVANS, Mark y DAVIES, Jonathan. 1999. "Understanding Policy Transfer: a Multi-Level, Multi-Disciplinary Perspective”, Public Administration 77, 2: 361-385. 


\section{Bibliografia}

GILARDI, Fabrizio. 2003. "Spurious and Symbolic diffusion of Independent Regulatory Agencies in Western Europe". Paper presentado en la conferencia The Internationalization of Regulatory Reforms, Berkeley University, 25-26 Abril 2003.

GraY, Virginia. 1973. "Innovation in the States: A diffusion Study". American Political Science Review 67, 4: 1174-1185.

Hall, Peter A. 1989. The Political Power of Economic Ideas: Keynesianism across Nations. Princeton/New York:Princeton University Press.

HaverLand, Markus. 2000. "National Adaptations to European Integration: The Importance of Institutional Veto Points". International Public Policy 20, 1: 83103.

HeClo, Hugh. 1974. Modern Social Politics in Britain and Sweden. From Relief to Income Maintenance. New Haven: Yale University Press.

Heinelt, Hubert y Hooghe, Liesbet. 1996. "Multilevel governance in the European Union and the Structural Fund", en Policy Networks and European Structural Funds, Heinelt et. al. (ed), Avebury: Ashgate Publishing Ltd.

HoberG, G. 1991. "Sleeping With an Elephant: The American Influence on Canadian Environmental Regulations”. Journal of Public Policy 11, 1.

Kennet, Patricia. 2001. Comparative Social Policy: Theory and Research. Buckingham/Philadelphia: Open University Press.

KeRn, Kristine, KISSLING-NäF, Ingrid and Landmann, Ute Mauch Corine. 2001. Policy Convergence and Policy Diffusion by Governmental and Non-governmental Institutions-An international comparison of Eco-labelling systems. Discussion paper, FS II 01-305, Wissenschafiszentrum Berlin Für Sozialforshung, Berlin.

Kingdon, John. 1996. Agendas, Alternatives and Public Policies. New York: HarperCollins.

KnILL, Christoph. 1998. European Policies. The Importance of National Administrative Traditions. Journal of Public Policies 18: 1-28.

Knill, Christoph and LeHMkUHL, Dirk. 2002. The national impact of European Union regulatory policies: three Europeanization mechanisms", European Journal of Political Research, 41: 255-280.

KOHLER-KoCH, Beate. 2002. European Networks and Ideas: Changing National Policies?, en European Integration online Papers 6(6), http://eiop.or.at/eiop/texte/2002-006a.html

Majone, Giandomenico. 1991. "Coss-National Sources of Regulatory Policymaking in Europe and in the United States". Journal of Public Policy 11, 1: 79-106.

MeSEGUER, Covadonga. 2003. "The diffusion of privatization in OECD countries: what role for learning?". Paper presentado en la conferencia The Internationalization of Regulatory Reforms, Berkeley University, 25-26 Abril 2003.

MinTrOM, Michael y VerGari, Sandra. 1998. "Policy Networks and Innovation Diffusion: The case of State Education Reforms". The Journal of Politics 60, 1: 126-148.

Palloni, Alberto. 1998. "Theories and Models of Diffusion in Sociology". Working paper, Center for Demography and Ecology, University of WisconsinMadison.
Peters, Guy. 1998. "Policy Networks: Myth, Methaphor and Reality" en Comparing Policy Networks, Marsh, David (ed.), Buckingham/Philadelphia: Open University Press.

Pierson, Paul (ed.). 2001. The New Politics of the Welfare State. New York/Oxford: Oxford University Press.

RADAELLI, Claudio. 2000. "Policy Transfer in the European Union: institutional isomorphism as a Source of Legitimacy", en Governance: an International Journal of Policy and Administration 13, 1, 25-43.

Ragin, Charles. 1987. The Comparative Method. Moving Beyond Qualitative and Quantitative Strategies. Berkeley:University of California Press.

RoberTson, David Brian. 1991. "Political Conflict and Lesson-Drawing". Journal of Public Policy 11, 1: 31-54.

Rogers, Everett. 1995. The Diffusion of Innovations. 4a edición. New York: The Free Press.

Rose, Richard. 1991. "What is Lesson-Drawing?" Journal of Public Policy 11, 1: 3-30.

Sabatier, Paul. 1991. "Towards Better Theories of the Policy Process". Political Science and Politics, 24, 2: 147-156.

Sabatier, Paul and Jenkins-Smith, Hanks. 1993. Policy Change and Learning. An Advocacy Coalition Approach. Boulder:Westview Press.

Schneider, Volker and TenbÜCKen, Marc. 2003. "Divergent Convergence: The diffusion of Regulatory Reforms in the Telecommunications Sector". Paper presentado en la conferencia The Internationalization of Regulatory Reforms, Berkeley University 25-26 Abril 2003.

Simmons, Beth and ElKins, Zachary. 2003. "The globalization of Liberalization: Policy diffusion in the International Political Economy". Paper presentado en la conferencia The Internationalization of Regulatory Reforms, Berkeley University 25-26 Abril 2003.

SmelSer, Neil J. 1976. "Comparative Methods in Social Sciences". Englewood Cliffs: Prentice-Hall.

STONE, Diane. 1999. "Learning Lessons and Transferring Policy across Time, Space and Disciplines". Politics 19, 1:51-59.

StONE, Diane. 2003. "Learning Lessons, Policy Transfer and the International Diffusion of Policy Ideas". Paper presentado en la conferencia Political Economy of Policy Transfer, Learning and Convergence, Tulane University, Abril 2003.

WALKER, Jack. 1969. "The Diffusion of Innovations among the American States". American Political Science Review 63: 880-899.

WeYLAnd, Kurt. 2002. "The Diffusion of Innovations. A Theoretical Analysis". Paper presentado en la Conferencia Anual de la American Political Science Association, Boston, septiembre 2002. 
